\title{
REVIEW
}

\section{FDG-PET/CT for cancer management}

\author{
Hideki Otsuka, Naomi Morita, Kyo Yamashita, and Hiromu Nishitani \\ Department of Radiology, Institute of Health Biosciences, The University of Tokushima, Graduate \\ School, Tokushima, Japan
}

\begin{abstract}
FDG-PET/CT is increasingly being used for staging, restaging, and treatment monitoring for cancer patients. The introduction of a PET/CT system enables both morphological and metabolic imaging to be performed in a single session. Knowledge of the normal physiologic distribution of FDG and an understanding of the clinical indications and limitations of PET/CT enable accurate diagnosis and thus a better level of care for patients. J. Med. Invest. 54 : 195-199, August, 2007
\end{abstract}

Keywords : FDG, PET/CT, oncology, cancer staging

\section{INTRODUCTION}

Functional images using PET (positron emission tomography) and anatomical images using CT (computed tomography) have traditionally been acquired in separate sessions. It has been hypothesized that combining these images leads to improved diagnosis and thereby better patient care. The introduction of a PET/CT system enables both morphological and metabolic imaging to be performed in a single session. The PET/CT system has become widespread and plays an important role in clinical oncology (1-5).

\section{RADIONUCLIDE}

The most commonly used radioisotope for PET imaging is fluorine-18, which has a half-life of 109.8 minutes and is cyclotron-produced. Fluorine-18 can be substituted for hydrogen atoms and is most commonly used as FDG (fluoro-deoxy-glucose). FDG uptake reflects glucose metabolism and is based on the enhanced glycolysis associated with malignant

Received for publication January 30, 2007 ; accepted February 16, 2007.

Address correspondence and reprint requests to Hideki Otsuka, Department of Radiology, Institute of Health Biosciences, The University of Tokushima, Graduate School, Kuramoto-cho, Tokushima 770-8503, Japan and Fax : +81-88-633-7174. cells. In malignant cells, the glucose membrane transporter is overexpressed and upregulation of enzymecontrolling glycolytic pathways occurs. Once taken up into the cell, FDG is a substrate for hexokinase, the first enzyme of glycolysis, and is phosphorylated to FDG-6-phospate. Its metabolic activity ceases and it remains in the cells (metabolic trapping) ; thus, its concentration accurately reflects the glycolytic activity of exogenous glucose. FDG uptake is dependent on many factors, including proliferative activity, the number of viable cells, histological grading, and inflammatory reactions.

\section{FDG-PET/CT SCAN}

After fasting for 6 hours, the patient is administered $3.7 \mathrm{MBq} / \mathrm{kg}$ of FDG intravenously using an automatic injection system. The blood glucose level is checked before injection and confirmed to be less than $150 \mathrm{mg} / \mathrm{dl}$. The patient lies quietly on a bed in a separate booth during the uptake phase. Water intake and frequent voiding is recommended, with the bladder emptied immediately prior to scanning. The PET/CT scan begins 1 hour after injection of FDG. Before scanning, the patient is required to remove all metal objects from the body (bracelets, dental braces, etc.) that could cause streak artifacts on the CT images. The patient is positioned on the examination table with his/her arms by his/her 
side, and breathes freely throughout the examination. The PET/CT scan begins with a topogram that is used to define the axial range of the body. At our institute, the range is from the top of the head to the middle of the thigh. The axial ranges of the PET and CT components of the combined examinations are matched to ensure fully quantitative attenuation correction of the emission data ; CT data are used to confirm anatomical location and for attenuation correction. In a traditional stand-alone PET system, a transmission scan would be obtained for attenuation correction, requiring extra time. Once the CT scan is complete, PET acquisition begins in the caudal-cranial direction. The entire examination is completed in 30 minutes or less.

\section{PHYSIOLOGICAL FDG UPTAKE}

Fig. 1 shows normal FDG distribution in the brain (gray matter), pharynx, larynx, thyroid, myocardium, liver, spleen, urinary tract, muscles, bowel,

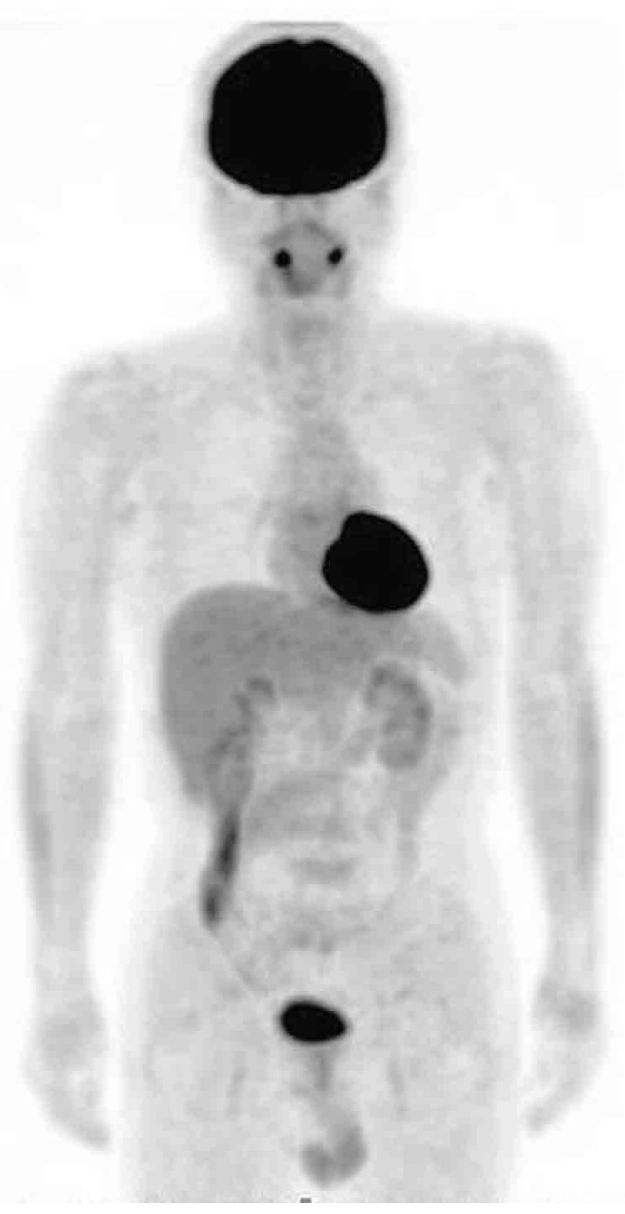

Fig. 1 PET image of 45 year-old male. Normal FDG distribution is seen in the brain (gray matter), pharynx, larynx, thyroid, myocardium, liver, spleen, urinary tract, muscles, bowels and genitalia. and genitalia. Distribution varies with the individual. Brain parenchyma utilizes glucose as an energy source and usually shows very intense uptake. The relative uptake in the brain is lower when the blood glucose level is high because FDG and blood glucose compete for intravascular space (Fig. 2). Myocardial uptake varies from absent to intense. The uptake can vary even for the same patient at different times. Myocardial uptake increases in the presence of insulin. Liver uptake is usually homogenous and mildly intense, although it may have a mottled appearance. Mottled uptake may limit the detection of small lesions. Uptake in the alimentary tract can be observed in the stomach, small bowel, and colon. Bowel uptake can vary even in the same patient on different occasions, and can be recognized by location and a tubular uptake pattern. Small lesions can be masked ; focal uptake mimics tumor. CT imaging or delayed PET scanning 2 hours after FDG injection may be helpful to distinguish normal physiological uptake from tumor. Intestinal inflammation can result in dense FDG uptake. Muscular uptake varies with insulin level and exercise ; patients must avoid strenuous exercise on the day before their PET scan. Bone marrow uptake is generally low-level. Patients with anemia or those undergoing chemotherapy with colony-stimulating factors can show diffuse increased uptake that reflects activated or recovering bone marrow.

\section{CASE PRESENTATION}

In clinical oncology, PET/CT is used to differentiate between malignant and benign lesions, determine the stage of cancer, monitor the effects of therapy, and detect recurrence. Indications for PET/CT examination include brain tumors, head and neck cancers, lung cancer, breast cancer, esophageal cancer, colon cancer, pancreatic cancer, metastatic liver tumors, uterine cancer, ovarian cancer, malignant lymphoma, malignant melanoma, and cancer of unknown primary origin.

A 75-year-old male patient with lung cancer underwent PET/CT for staging. The primary lesion showed intense FDG uptake with hilar and mediastinal lymph node metastasis. No distant metastasis was detected, and this patient was staged T2N2M0, stage IIIA (Fig. 3).

A 47-year-old male lymphoma patient was examined for staging and restaging after four courses of chemotherapy (Fig. 4). The patient was diagnosed 
as stage IIIa by PET findings. After chemotherapy, abnormal intense uptake decreased or disappeared. The regimen was effective and this patient was considered to be in complete remission.

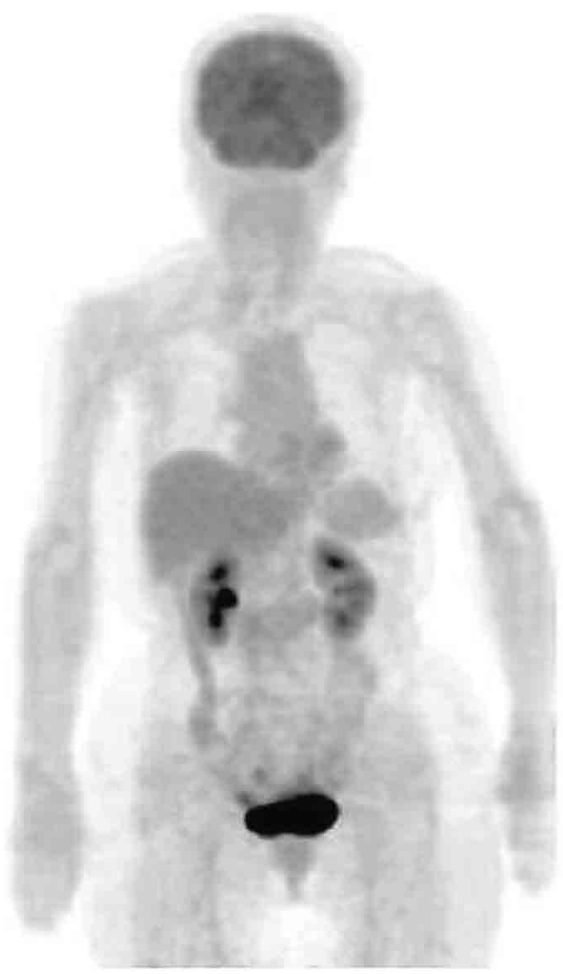

A 64-year-old female ovarian cancer patient was examined for tumor marker elevation. Routine CT examination was unable to distinguish whether the lesion was recurrent or metastatic ; however, FDG-

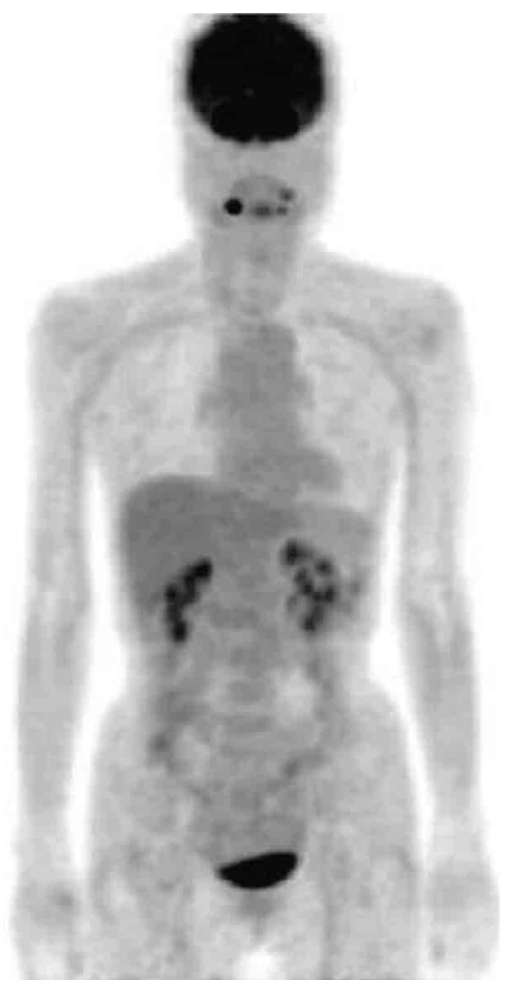

Fig. 2 Variations of brain FDG uptake. a) blood sugar level $221 \mathrm{mg} / \mathrm{dl}$, b) blood sugar level $100 \mathrm{mg} / \mathrm{dl}$. The relative uptake in the brain is lower when the blood glucose level is high because FDG and blood glucose compete in vascular space

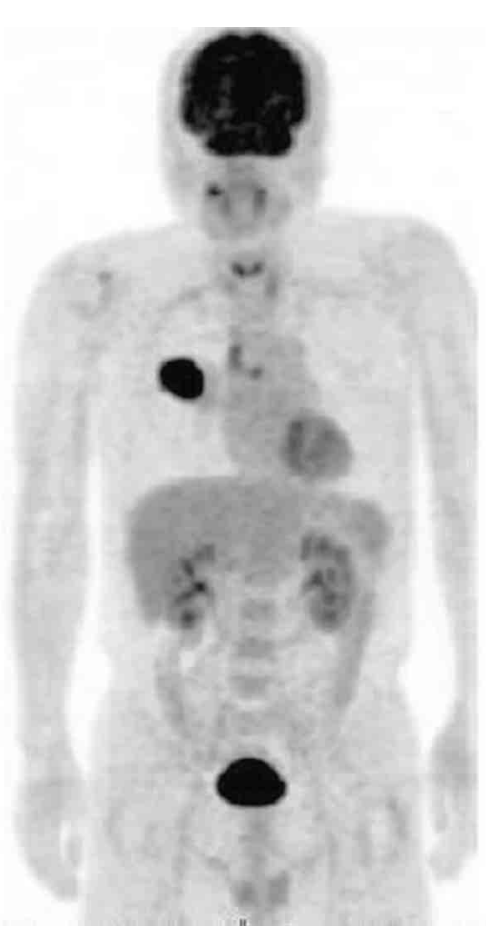

a) b)

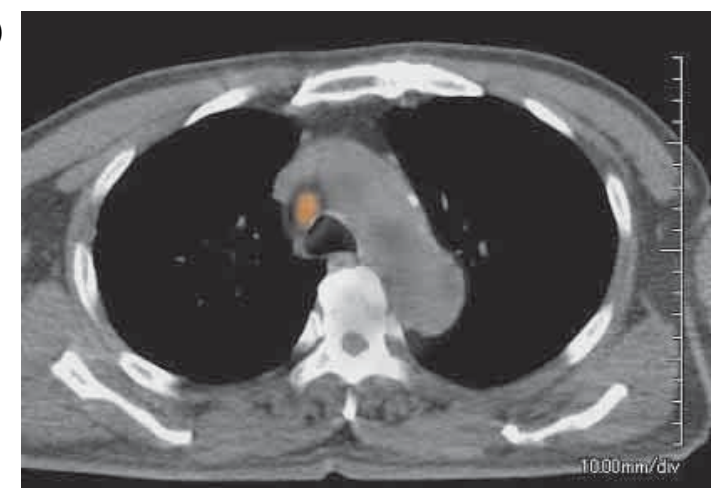

c)

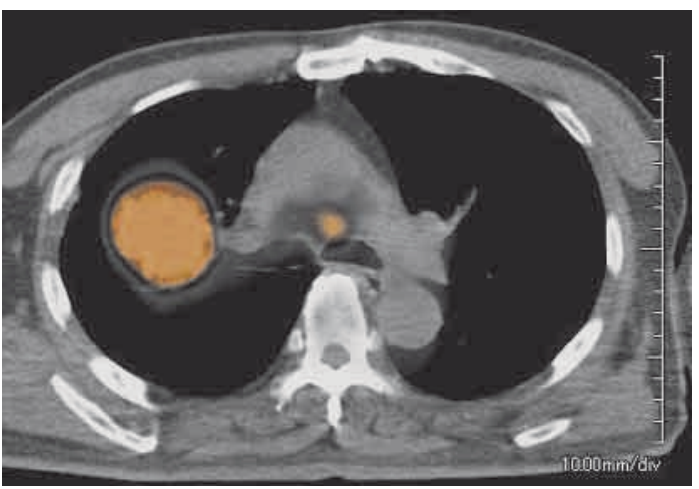

Fig. 3 Lung cancer. A 75-year-old male patient with lung cancer was indicated PET/CT for staging. Primary lesion showed intense FDG uptake with hilar and mediastinal lymph nodes metastasis. No distant metastasis is detected and this patient can be staged T2 N2M0, stage IIIA 
PET/CT demonstrated a recurrent lesion in the pelvis. The lesion showed high nodular FDG uptake corresponding to a soft tissue mass that was difficult to differentiate from the surrounding normal struc-

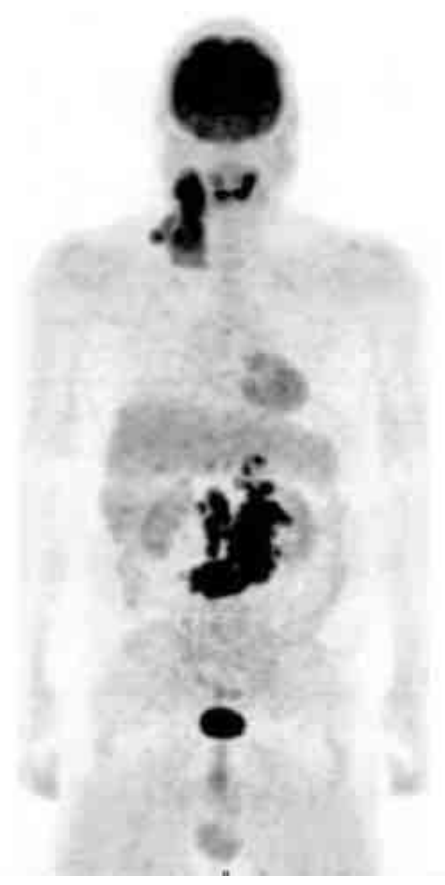

a) tures (Fig. 5).

High-density structures such as metallic dental dentures and cardiac pacemakers can cause artifacts on CT (Fig. 6). In the PET/CT system, CT data are

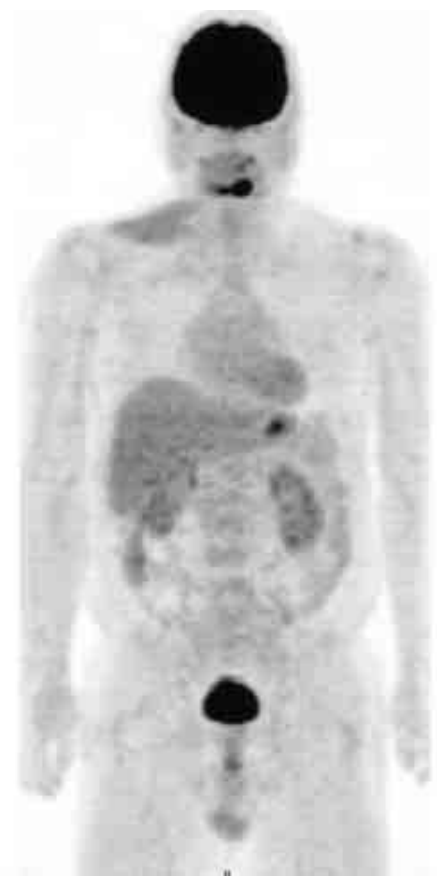

b)

Fig. 4 Lymphoma. A 47-year-old male lymphoma patient was examined for staging and restaging. a) The patient was diagnosed as stage IIIa by PET findings. b) After 4 courses of chemotherapy, abnormal intense uptake decreased or disappeared. The regimen was effective and this patient was considered to be in complete remission. Diffuse low uptake in the right shoulder corresponds to physiological muscular uptake.

a)



b)

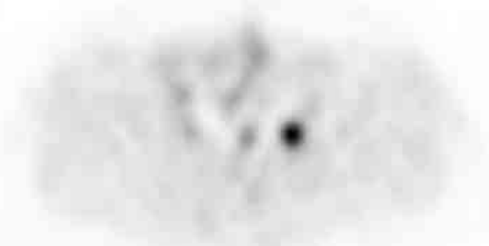

d)
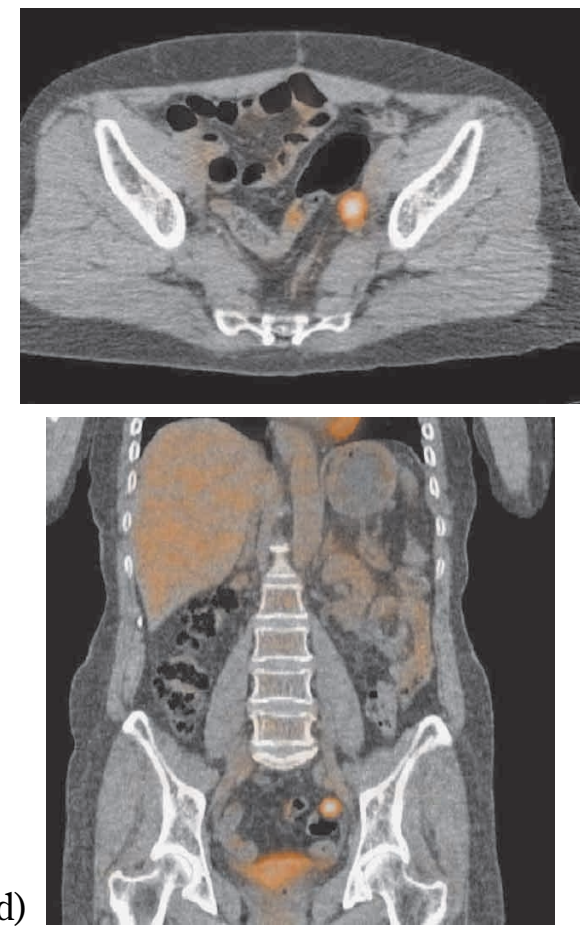

Fig. 5 Recurrence of ovarian cancer. A 65-year-old female ovarian cancer patient was examined for tumor marker elevation. Routine CT examination was unable to distinguish whether the lesion was recurrent or metastatic; however, FDG-PET/CT demonstrated a recurrent lesion in the pelvis. The lesion showed high nodular FDG uptake corresponding to a soft tissue mass that was difficult to differentiate from the surrounding normal structures. 

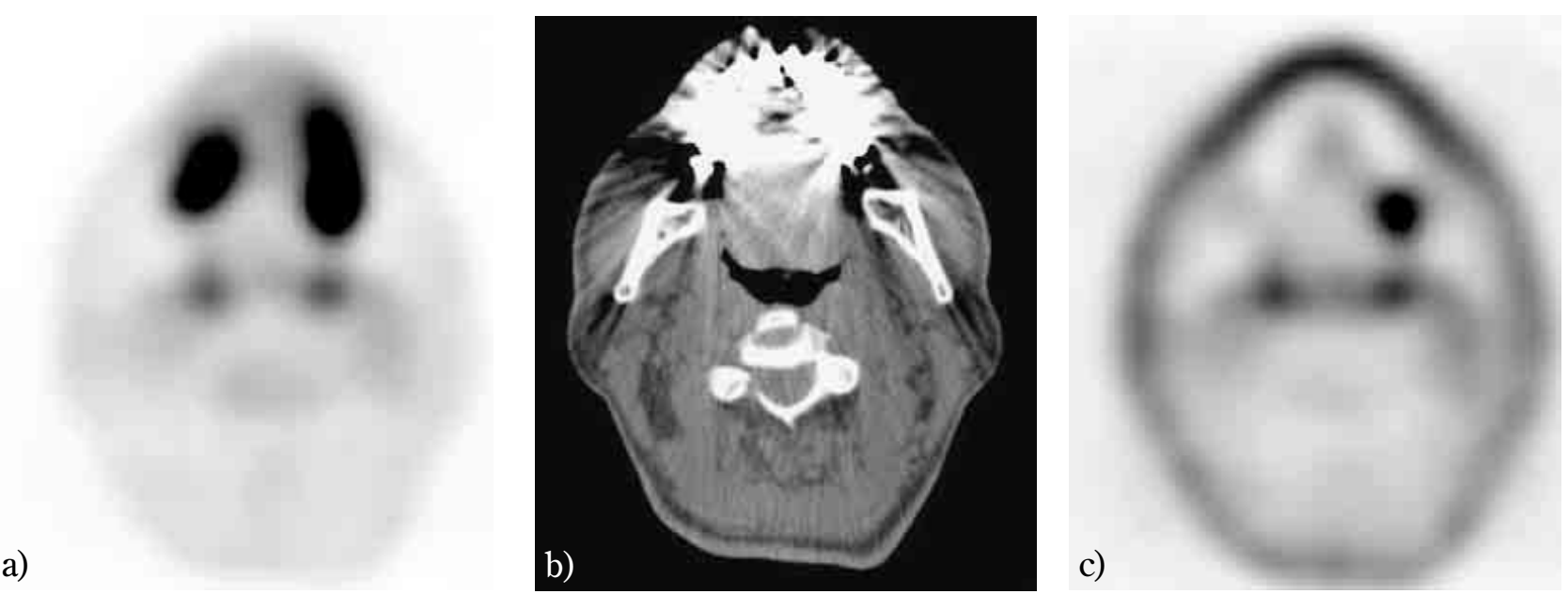

Fig. 6 Dental denture artifact. a) Attenuation-corrected PET image, b) CT image, c) non attenuation-corrected image. The energy of positron is much higher than X ray, thus PET data is overestimated. Tongue cancer is unclear in attenuation-corrected PET image, but it clearly demonstrated in non attenuation-corrected PET image.

used for attenuation correction. As positron energy is much more intense than $\mathrm{X}$ ray energy, PET data are overestimated. It is necessary to review nonattenuation-corrected PET images in image interpretation. Tongue cancer is unclear in attenuationcorrected PET images but is clearly demonstrated in non-attenuation-corrected PET images.

\section{SUMMARY}

PET/CT enables metabolic and morphologic images to be acquired in a single session. The success of PET/CT depends on the accurate interpretation of detailed clinical history, with possible sources being the referring physician, the patient themselves, and family members. FDG uptake depends on tumor size, malignant cell density, and histological grading. Knowledge of the normal physiologic distribution of FDG and an understanding of the clinical indications and limitations of PET/CT enable accurate diagnosis and thus a better level of care for patients.

\section{ACKNOWLEDGEMENTS}

I would like to thank all of the staff at our imaging center for their valued assistance with this study.

\section{REFERENCES}

1. Chung HH, Kang WJ, Kim JW, Park NH, Song YS, Chung JK, Kang SB, Lee HP : Characterization of surgically transposed ovaries in integrated PET/CT scan in patients with cervical cancer. Acta Obstet Gynecol Scand 86(1) : 8893, 2007

2. Czernin J, Allen-Auerbach M, Schelbert HR. : Improvements in Cancer Staging with PET/CT : Literature-Based Evidence as of September 2006. J Nucl Med 48 (Suppl 1) : 78S-88S, 2007

3. Jinnouchi S, Tanabe H, Yoshida T, Ochiai R: FDG-PET manual. Innervision, Tokyo, 2004

4. Inoue T, Nakamoto Y : Available Knowledge for Clinical Practice in FDG-PET oncology. Jpn J of Diagnostic Imaging 25(9) : 1096-1159, 2005

5. Moriyama N, Magashima T, Kubota K, Tatsumi $\mathrm{M}$ : Diagnosis of primary and wetastate lesions using PET. Clinical Imaginology 21(12) : 12581323, 2005 